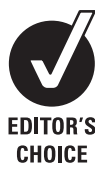

${ }^{1}$ Department of Public Health, Erasmus MC, Rotterdam, the Netherlands; ${ }^{2}$ AStri Research and Consultancy Group, Leiden, the Netherlands

Correspondence to: Alex Burdorf, Erasmus MC Department of Public Health, PO Box 2040, 3000 CA Rotterdam, The Netherlands; a.burdorf@ erasmusmc.nl

Accepted 17 October 2008 Published Online First 18 November 2008

\title{
The effects of work-related and individual factors on the Work Ability Index: a systematic review
}

\author{
T I J van den Berg, ${ }^{1}$ L A M Elders, ${ }^{1}$ B C H de Zwart, ${ }^{2}$ A Burdorf ${ }^{1}$
}

\section{ABSTRACT}

This paper systematically reviews the scientific literature on the effects of individual and work-related factors on the Work Ability Index (WAI)

Studies on work ability published from 1985 to 2006 were identified through a structured search in PubMed, and Web of Science. Studies were included if the WAI was used as measure of work ability and if quantitative information was presented on determinants of work ability.

In total, 20 studies were included with 14 cross-sectional studies and six longitudinal studies. Factors associated with poor work ability, as defined by WAI, were lack of leisure-time vigorous physical activity, poor musculoskeletal capacity, older age, obesity, high mental work demands, lack of autonomy, poor physical work environment, and high physical work load.

The WAI is associated with individual characteristics, lifestyle, demands at work, and physical condition. This multifactorial nature of work ability should be taken into account in health promotion programmes aimed at maintaining and promoting the participation of the labour force and improvement of the performance at work.

Most Western countries with an ageing population face the challenge of a need to increase work participation, especially at older age. Governmental policies are implemented to increase the age of full retirement in order to balance the ratio of employed over dependent persons. ${ }^{1}$ Yet, in most countries the average age of permanent departure from paid labour is well below the statutory pension age, ${ }^{2}$ so there is a need to develop interventions that will facilitate workers to be engaged in paid employment until pension age.

Ageing of workers is accompanied with changes in physical and mental capacities. However, individual differences are large and lifestyle factors such as physical activity in leisure time may substantially influence the balance between work capacity and work demands. ${ }^{3}$ Work demands that are not sufficiently attuned to physical and mental capacities of workers may increasingly cause health problems and subsequently displacement from the workforce. ${ }^{2}$ The contribution of (work-related) health problems to unemployment and early retirement among older workers is substantial. ${ }^{4}$

In order to increase work participation and prolong the working life among older workers the concept of work ability has been developed in the early 1980s in Finland, and was later adopted in various other European and Asian countries. According to Ilmarinen, ${ }^{5}$ work ability is built on the balance between a person's resources and work demands. The bases for work ability are health and functional capacity, but work ability is also determined by professional knowledge and competence (skills), values, attitudes, and motivation, and work itself.

Work ability has been measured in different ways. For example, by single questions asking respondents to range their current work ability on a 5- or 10-point scale. ${ }^{6}$ Moreover, work ability has been defined as not being on long-term sick leave, ${ }^{7}$ or in total days on sick leave during the last 12 months. ${ }^{8}$ Studies have shown that a poor work ability increased the risk of early retirement, ${ }^{9}$ longterm sickness absence, and work disability. ${ }^{10}$

The Work Ability Index (WAI) ${ }^{11}$ is by far the most used, and well-accepted instrument to measure work ability, as is demonstrated by its availability in 21 languages. Although several studies in different occupational settings have been conducted, there is a need for a systematic evaluation of the relative importance of workrelated and individual determinants of work ability, measured with the WAI. This knowledge of determinants of work ability is important to tailor interventions aimed at increasing work participation among elderly workers, and maintenance or improvement of the productivity performance at work. In this article the epidemiological data on determinants of work ability over the past 25 years have been reviewed. The aim of this systematic review is to identify the individual and work-related determinants of work ability, measured with the WAI among occupational populations.

\section{METHODS \\ Identification of the studies}

Relevant articles were identified by means of a computerised search of the bibliographical databases PubMed January 1985-December 2006, and Web of Science over the period January 1988December 2006. The following search string was used: "work ability". The search was restricted to studies published in the English language. The literature search identified 337 abstracts with 124 corresponding abstracts in both databases, resulting in 213 unique abstracts.

\section{Selection}

The initial selection of studies was performed by the first author (TB), and verified by the last author (AB). Studies were excluded if (a) the WAI was not applied to describe work ability in an occupational population; and/or (b) no quantitative information on associations between individual and work-related factors and work ability was presented. The WAI is an assessment of the ability 
of a worker to perform his/her job, taking into account the specific psychosocial and physical work-related factors, mental and physical capabilities, and health. The index consists of a questionnaire on physical and mental demands of an individual in relation to their work, diagnosed diseases, limitations in work due to disease, sick leave, work ability prognosis, and psychological resources. These seven dimensions are rated and the summative index ranges from 7 to 49 , which is classified into poor (7-27), moderate (28-36), good (37-43), and excellent (44-49) work ability. ${ }^{11}$

Based on title and abstract, 146 out of 213 abstracts (69\%) were discarded due to lack of any quantitative description of associations between individual and work-related determinants and the WAI. Another seven articles (3\%) did not use the WAI for measurement of work ability. Another four abstracts were duplicates and four abstracts did not have a full article. In total, 52 articles were retrieved for further review. Of these articles, 26 out of $52(50 \%)$ were excluded due to lack of quantitative information on associations between determinants and work ability, and another 9 (17\%) did not use the WAI. Thus, 17 $(33 \%)$ publications remained that met our selection criteria. ${ }^{12-28}$ One publication was included after an additional search in the references of the articles included for review. ${ }^{29}$ Since two publications reported the results of both a cross-sectional study and a follow-up study, in total 20 studies were included in this review. ${ }^{16} 22$

\section{Data extraction}

The data extraction on selected full articles comprised the study population, study design, research setting, outcome(s), determinants, confounders or effect modifiers, and estimates of effects (with $95 \%$ confidence intervals). Determinants of work ability, as defined by the WAI were categorised as individual characteristics, and work-related factors. Individual characteristics were demographic variables, physical condition, and lifestyle factors. Workrelated factors were physical work demands, and psychosocial work demands. Some studies reported also on other determinants that are partly included in the WAI measurement itself, for example, health complaints, and work satisfaction. Due to this dependency between determinant and WAI, these determinants were not evaluated in this systematic review. Data extraction was performed by one author according to a standardised format (TB) and extracted data were reviewed by another author on consistency and completeness $(A B)$. In case of doubt, data were discussed until agreement was reached $(T B, A B)$.

The analysis focused on measures of association, expressed by, for example an odds ratio (OR), or a regression coefficient. Whenever possible the measure of association was retrieved from the original article, together with the variables that were adjusted for in the statistical analysis. In case this information was not present, available raw data in a $2 \times 2$ table were used to calculate an $\mathrm{OR}$ and confidence interval.

\section{Classification of associations}

In this review, three types of statistical associations are distinguished. The association is described as positive when a determinant is statistically significantly associated with an increased risk for a poor WAI or a reduced WAI. The association is described as negative when a determinant is statistically significantly associated with a decreased risk for a poor WAI or a reduced WAI. In a null association no significant association was found between the determinant and WAI. In order to increase the comparability of the studies, the direction of the association presented in the original article was adjusted when needed to assure that an OR above 1 or a positive regression coefficient have a similar interpretation across all studies.

\section{Quality assessment}

The quality of the epidemiological studies (see table 1) was assessed by two reviewers ( $T B$ and $A B$ ) using a standardised form based on seven items in a modified version of the guidelines for methodological quality assessment of the Dutch Cochrane Centre ${ }^{30}$ :

- Study population; the characteristics of the population, at least age, sex and occupation, should be described in detail;

- Sample size and statistical power; the number of subjects should at least be 10 times the number of covariates;

- Response; the response at baseline should be at least 70\%;

- Selection bias; substantial selection bias is not likely to be present;

- Measurement error: substantial misclassification in determinants is most likely not present;

- The assessment of the determinants should be blinded to the WAI measurement;

- Confounding; the analysis should be adjusted for confounders.

Each criterion was rated when applicable, with a score of 1 being "sufficiently met", a score of 0 being "not sufficiently met", and a question mark when information was lacking to rate this item. The total quality score was rated from 0 to 7 .

\section{RESULTS}

In total, determinants of work ability were reported in 14 crosssectional studies, and six longitudinal studies. Individual characteristics were addressed in 18 studies and work-related characteristics in nine studies. Occupations most studied in relation to work ability were (Finnish) municipal workers and care givers. In fact, all longitudinal studies regarding workrelated characteristics were carried out among Finnish municipal workers.

The majority of the studies focused on a poor WAI as a dichotomous outcome, either defined by specific threshold level (mostly 37 ), or lowest $25 \%$ or $15 \%$ percentiles (tables $2-5$ ).

\section{Individual characteristics}

The demographic factor most studied was age (seven studies) (tables 2 and 3). Four out of seven studies reported a decreased WAI with older age, ${ }^{15} 172024$ two studies demonstrated no association $^{26}{ }^{29}$ and one study found a higher risk for a poor WAI among younger workers. ${ }^{14}$ Sex $(n=2)^{17} 29$ was not associated with WAI, whereas a lower education was associated with a lower WAI in one study ${ }^{17}$ and had no effect in another study. ${ }^{29}$ Being a sole breadwinner, and degradation in economic position were associated with lower WAI, ${ }^{14}{ }^{27}$ whereas no relation was observed for low income. ${ }^{29}$ Four studies reported on other individual characteristics. A lower WAI was associated with hard life situation outside work, ${ }^{20}$ raising underage children, ${ }^{14}$ and low self-confidence, ${ }^{23}$ and not significantly associated with marital status. ${ }^{29}$

One out of three studies found a positive association between a better cardiorespiratory fitness, expressed by maximum oxygen uptake, and a higher WAI. ${ }^{15}$ All four studies on poor musculoskeletal capacity reported a significant association with a poor WAI with risk estimates varying from 6.4 to $9.1 .{ }^{13} 181923$ Poor functional balance in home care workers was associated with poor WAI ${ }^{19}$ whereas this association was not observed in 
Table 1 Results of the quality assessment of 20 selected studies with quantitative information on associations between individual and work-related factors and work ability, as measured with the Work Ability Index

\begin{tabular}{|c|c|c|c|c|c|c|c|c|c|}
\hline Study (first author) & Design & $\begin{array}{l}\text { Quality score } \\
(0-7)\end{array}$ & $\begin{array}{l}\text { Study } \\
\text { population }\end{array}$ & $\begin{array}{l}\text { Sample } \\
\text { size }\end{array}$ & Response & $\begin{array}{l}\text { Selection } \\
\text { bias }\end{array}$ & $\begin{array}{l}\text { Measurement } \\
\text { error }\end{array}$ & Blinding & Confounding \\
\hline Aittomaki et al $2003^{12}$ & Cross-sectional & 6 & 1 & 1 & 0 & 1 & 1 & 1 & 1 \\
\hline Eskelinen et al $1991^{13}$ & Cross-sectional & 4 & 1 & 1 & $?$ & 0 & 1 & 0 & 1 \\
\hline Fischer et al $2006^{14}$ & Cross-sectional & 5 & 1 & 0 & 1 & 1 & 0 & 1 & 1 \\
\hline Goedhard et al $1998^{15}$ & Cross-sectional & 2 & 0 & 1 & $?$ & $?$ & 1 & $?$ & 0 \\
\hline Kaleta et al $2006^{28}$ & Cross-sectional & 3 & 0 & 1 & 0 & 1 & $?$ & 1 & 1 \\
\hline Laitinen et al $2005^{16}$ & Cross-sectional & 5 & 1 & 1 & 0 & 1 & 1 & $?$ & 1 \\
\hline Laitinen et al $2005^{16}$ & Prospective cohort & 5 & 1 & 1 & 0 & 1 & 0 & 1 & 1 \\
\hline Martinez et al $2006^{29}$ & Cross-sectional & 5 & 1 & 1 & 1 & 1 & 0 & 1 & 0 \\
\hline Monteiro et al $2006^{17}$ & Cross-sectional & 6 & 1 & 1 & 1 & 1 & ? & 1 & 1 \\
\hline Nygard et al $1991^{18}$ & Cross-sectional & 4 & 1 & 1 & $?$ & 1 & 1 & 0 & 0 \\
\hline Pohjonen 2001 $a^{19}$ & Prospective cohort & 5 & 1 & 1 & $?$ & $?$ & 1 & 1 & 1 \\
\hline Pohjonen 2001b & Cross-sectional & 6 & 1 & 1 & 1 & 1 & 0 & 1 & 1 \\
\hline Pranjic et al $2006^{21}$ & Cross-sectional & 5 & 1 & 1 & 1 & 1 & 0 & 1 & 0 \\
\hline Punakallio et al $2004^{22}$ & Cross-sectional & 6 & 1 & 1 & 1 & 1 & 1 & $?$ & 1 \\
\hline Punakallio et al $2004^{22}$ & Prospective cohort & 7 & 1 & 1 & 1 & 1 & 1 & 1 & 1 \\
\hline $\begin{array}{l}\text { Sjogren-Ronka et al } \\
2002^{23}\end{array}$ & Cross-sectional & 6 & 1 & 1 & 1 & 1 & 1 & 0 & 1 \\
\hline Tuomi et al $1991^{24}$ & Prospective cohort & 5 & 1 & 1 & $?$ & 1 & 0 & 1 & 1 \\
\hline Tuomi et al $1997^{26}$ & Prospective cohort & 6 & 1 & 1 & 1 & 1 & 0 & 1 & 1 \\
\hline Tuomi et al $2001^{25}$ & Cross-sectional & 5 & 1 & 1 & 1 & 0 & 0 & 1 & 1 \\
\hline Tuomi et al $2004^{27}$ & Prospective cohort & 5 & 1 & 1 & 0 & 1 & 0 & 1 & 1 \\
\hline
\end{tabular}

1, "sufficiently met"; 0, "not sufficiently met"; '?', information was lacking.

two studies among fire fighters. ${ }^{22}$ Both studies on general cognitive mental performance showed no significant associations. ${ }^{13} 18$

Overweight was positively associated with a poor WAI in four out of seven studies. ${ }^{14} 192425$ Lack of leisure-time physical activity was associated with a lower WAI in four out of five studies. ${ }^{25-28}$ In one study, smoking was associated with lower WAI, ${ }^{24}$ whereas in two studies no significant association was found. ${ }^{2528}$ One study reported a positive effect of alcohol drinkers versus teetotalers on WAI. ${ }^{25}$ In one study a diet with low fibre intake was reported with an OR of 27.6 for a poor WAI. ${ }^{28}$

\section{Work-related factors}

A large variety of psychosocial factors at work were addressed, varying from poor management to satisfaction with supervisor (tables 4 and 5). Five out of seven studies reported a positive association between high mental work demands and a poor WAI, ${ }^{21}{ }^{23-25} 27$ whereas among home care workers, ${ }^{20}$ and care givers $^{14}$ no significant associations were reported. Three out of four studies reported a positive association with a poor WAI for lack of autonomy, ${ }^{20}{ }^{25}{ }^{27}$ whereas one study failed to corroborate this association. ${ }^{26}$ High physical demands, such as increased muscular work, poor work postures, and poor ergonomic conditions were positively associated with a lower WAI in four out of seven studies. ${ }^{202426}$ Regarding the physical work environment, two out of four studies reported a lower WAI with thermal discomfort and poor physical climate, ${ }^{24}{ }^{25}$ whereas another two studies did not find any association. ${ }^{14} 26$

\section{Quality rating}

Quality scores ranged from 2 to 7 (table 1). A low response at baseline (less than 70\%), and measurement error were most present shortcomings in quality. There were no statistically significant differences in quality score for design, type of determinant (individual vs work related), and whether a significant association was reported or not. Due to the large heterogeneity in definitions of determinants, a meta-analysis was not possible.

\section{DISCUSSION}

This review showed that factors associated with decreased work ability, were lack of leisure-time vigorous physical activity, poor musculoskeletal capacity, older age, obesity, and high physical and psychosocial work demands. No conclusions can be drawn regarding the relative importance of the determinants, because of the large heterogeneity in study characteristics (study populations, sample size, definition of determinants).

\section{Limitations}

This systematic review has some limitations. The literature search may not be comprehensive enough because publications in languages other than English were not included, and the search was limited to two computer-based bibliographic databases. The search in Web of Science resulted in an additional 51 articles relative to PubMed, but all of these were finally excluded. However, it cannot be ruled out that relevant publications would have been identified when using additional databases.

In the selection of relevant literature, 16 abstracts (8\%) were excluded, since work ability was used as a generic term without a clear method of measurement. These studies merely focused on generic work ability without measuring. In the full review of selected articles, nine articles (18\%) were excluded since work ability was not quantified $(n=1)$ or measured differently from the WAI $(n=8)$, for example using one question on current work ability with differing scales $(n=4)$ or based on the number of sick leave days $(n=2)$. This latter finding suggests that the WAI is indeed the most often used instrument to quantify the work ability in occupational populations.

An important limitation is that the majority of studies were of cross-sectional design and, as a consequence, causality cannot be determined. A clear example is the study reporting on a negative association between job retraining and a poor WAI. ${ }^{31}$ It 
Table 2 Associations between individual determinants and Work Ability Index (WAI) in cross-sectional epidemiological studies among occupational populations

\begin{tabular}{|c|c|c|c|c|c|}
\hline Authors & Study population & WAI & Determinant & Association (95\% CI) & Adjustments* \\
\hline \multirow[t]{4}{*}{ Eskelinen et al $1991^{13}$} & \multirow[t]{2}{*}{$\begin{array}{l}89 \text { municipal workers }(M), \\
\text { mean age } 53 \text { years }\end{array}$} & \multirow[t]{2}{*}{$\begin{array}{l}\text { Poor WAI (lowest } \\
25 \% \text { ) }\end{array}$} & $\begin{array}{l}\text { Reduced cardiorespiratory } \\
\text { capacity }\end{array}$ & OR 1.85 (0.42 to 8.19$)$ & None \\
\hline & & & $\begin{array}{l}\text { Impaired mental performance } \\
\text { level }\end{array}$ & OR 1.75 (0.62 to 4.96$)$ & None \\
\hline & \multirow[t]{2}{*}{$\begin{array}{l}85 \text { municipal workers }(F) \text {, } \\
\text { mean age } 52 \text { years }\end{array}$} & \multirow[t]{2}{*}{$\begin{array}{l}\text { Poor WAI (lowest } \\
25 \%)\end{array}$} & $\begin{array}{l}\text { Impaired musculoskeletal } \\
\text { capacity }\end{array}$ & OR 9.12 (2.96 to 28.07$)$ & None \\
\hline & & & $\begin{array}{l}\text { Impaired mental performance } \\
\text { level }\end{array}$ & OR 1.65 (0.56 to 4.85$)$ & None \\
\hline \multirow[t]{4}{*}{ Fischer et al $2006^{14}$} & \multirow[t]{4}{*}{$\begin{array}{l}696 \text { care givers, mean age } \\
34.9 \text { years }\end{array}$} & \multirow[t]{4}{*}{ WAI $<37$} & $\begin{array}{l}\text { Sole breadwinner versus } \\
\text { double income }\end{array}$ & OR 1.92 (1.32 to 2.81$)$ & $1 \mathrm{~A}, \mathrm{E} 3 \mathrm{~A} 4 \mathrm{C} 5 \mathrm{~A}, \mathrm{~B}$ \\
\hline & & & Raising underage children & OR 1.56 (1.06 to 2.29$)$ & $1 A, D 3 A 4 C 5 A, B$ \\
\hline & & & Age $\geqslant 40$ years & OR $0.71(0.47$ to 0.97$)$ & 1D, E 3A 4C 5A, B \\
\hline & & & $\mathrm{BMI} \geqslant 30 \mathrm{~kg} / \mathrm{m}^{2}$ & OR 2.71 (1.55 to 4.77$)$ & $1 \mathrm{~A}, \mathrm{D}, \mathrm{E} 4 \mathrm{C} 5 \mathrm{~A}, \mathrm{~B}$ \\
\hline \multirow[t]{2}{*}{ Goedhard et al $1998^{15}$} & \multirow{2}{*}{$\begin{array}{l}141 \text { workers, mean age } \\
39.5 \text { years }\end{array}$} & \multirow[t]{2}{*}{ WAI 7-49 } & Age (years) & $\beta-0.28$ (Sign.) & None \\
\hline & & & $\mathrm{VO}_{2 \max }(\mathrm{ml} / \mathrm{kg} / \mathrm{min})$ & $\beta 0.17$ (Sign.) & Sickness claims (/year) \\
\hline \multirow[t]{7}{*}{ Kaleta et al $2006^{28}$} & \multirow{4}{*}{$\begin{array}{l}94 \text { workers }(M), \text { mean age } \\
43.5 \text { years }\end{array}$} & \multirow[t]{4}{*}{ WAI $<37$} & $\mathrm{BMI}>30 \mathrm{~kg} / \mathrm{m}^{2}$ & OR $0.29(0.03$ to 3.07$)$ & $3 B, C$ \\
\hline & & & Current smoker & OR $1.61(0.30$ to 8.60$)$ & $3 A, B$ \\
\hline & & & $\begin{array}{l}\text { LT physical activity } \\
<1000 \mathrm{kcal} / \text { week }\end{array}$ & OR 7.18 (1.10 to 31.09$)$ & $3 A, C$ \\
\hline & & & Fibre intake $<30 \mathrm{~g} /$ day & OR 27.63 (3.44 to 221.71$)$ & None \\
\hline & \multirow{3}{*}{$\begin{array}{l}93 \text { workers }(F) \text {, mean age } 42.3 \\
\text { years }\end{array}$} & \multirow[t]{3}{*}{ WAI $<37$} & $\mathrm{BMI}>30 \mathrm{~kg} / \mathrm{m}^{2}$ & OR $1.37(0.36$ to 8.15$)$ & $3 B, C$ \\
\hline & & & Current smoker & OR 14.84 (3.07 to 26.42 ) & $3 A, B$ \\
\hline & & & $\begin{array}{l}\text { LT physical activity } \\
<750 \mathrm{kcal} / \text { week }\end{array}$ & OR 2.70 (1.82 to 8.46$)$ & $3 A, C$ \\
\hline \multirow[t]{4}{*}{ Laitinen et al $2005^{16}$} & \multirow{2}{*}{$\begin{array}{l}2674 \text { Finnish workers (M), } \\
\text { mean age } 31 \text { years }\end{array}$} & \multirow{2}{*}{$\begin{array}{l}\text { Poor WAI (lowest } \\
15 \% \text { ) }\end{array}$} & $\mathrm{BMI} \geqslant 35.0 \mathrm{~kg} / \mathrm{m}^{2}$ & OR 1.00 (0.4 to 2.4$)$ & $1 \mathrm{C}, 3 \mathrm{C}$ \\
\hline & & & Waist-to-hip ratio $\geqslant 1.0$ & OR 1.80 (1.2 to 2.8$)$ & $1 \mathrm{C}, 3 \mathrm{C}$ \\
\hline & \multirow{2}{*}{$\begin{array}{l}2948 \text { Finnish workers }(F) \text {, } \\
\text { mean age } 31 \text { years }\end{array}$} & \multirow{2}{*}{$\begin{array}{l}\text { Poor WAI (lowest } \\
15 \%)\end{array}$} & $\mathrm{BMI} \geqslant 35.0 \mathrm{~kg} / \mathrm{m}^{2}$ & OR $2.70(1.7$ to 4.4$)$ & $1 \mathrm{C}, 3 \mathrm{C}$ \\
\hline & & & Waist-to-hip ratio $\geqslant 1.0$ & OR 2.10 (1.3 to 3.3$)$ & $1 \mathrm{C}, 3 \mathrm{C}$ \\
\hline \multirow{5}{*}{$\begin{array}{l}\text { Martinez and Latorre } \\
2006^{29}\end{array}$} & \multirow{5}{*}{$\begin{array}{l}224 \text { office workers, mean age } \\
34.7 \text { years }\end{array}$} & \multirow[t]{5}{*}{ WAI $<37$} & Age $\geqslant 40$ years & OR 0.84 (0.37 to 1.91$)$ & None \\
\hline & & & Female & OR 1.43 (0.67 to 2.99$)$ & None \\
\hline & & & Low income & OR 0.98 (0.47 to 2.03 ) & None \\
\hline & & & Low education & OR 1.09 (0.51 to 2.30$)$ & None \\
\hline & & & Not married & OR 1.12 (0.54 to 2.33 ) & None \\
\hline Monteiro et al $2006^{17}$ & 651 municipal workers & WAI $<36.5$ & Age group $\geqslant 55$ years & OR 1.9 (1.18 to 3.18$)$ & $1 B, C, E 3 B, C, A$ \\
\hline & & & Female & OR 1.3 (0.90 to 2.12$)$ & $1 \mathrm{~A}, \mathrm{C}, \mathrm{E} 3 \mathrm{~B}, \mathrm{C}, \mathrm{A}$ \\
\hline & & & Low education & OR 1.2 (1.01 to 1.55$)$ & $1 \mathrm{~A}, \mathrm{~B}, \mathrm{E} 3 \mathrm{~B}, \mathrm{C}, \mathrm{A}$ \\
\hline Nygard et al $1991^{18}$ & $\begin{array}{l}137 \text { municipal workers, mean } \\
\text { age } 55 \text { years }\end{array}$ & $\begin{array}{l}\text { Poor WAI (lowest } \\
25 \% \text { ) }\end{array}$ & $\begin{array}{l}\text { Poor trunk muscular } \\
\text { endurance }\end{array}$ & OR 6.39 (2.41 to 16.94$)$ & None \\
\hline & & & Impaired mental performance & OR 1.54 (0.61 to 3.92$)$ & None \\
\hline Pohjonen $2001 b^{20}$ & 636 home care workers $(F)$, & Decrease in WAI & Age $50-62$ versus $19-34$ & OR 3.57 (2.04 to 5.88 ) & 1C, E 3B 4A, B, C 5A \\
\hline & mean age 42.3 years & classification & $\begin{array}{l}\text { Hard life situation outside } \\
\text { work }\end{array}$ & OR 1.96 (1.03 to 3.75$)$ & $1 \mathrm{~A}, \mathrm{C} 3 \mathrm{~B} 4 \mathrm{~A}, \mathrm{~B}, \mathrm{C} 5 \mathrm{~A}$ \\
\hline & & & $\begin{array}{l}\text { LT physical activity }<1 \text { time a } \\
\text { week }\end{array}$ & OR 1.77 (0.88 to 3.55$)$ & None \\
\hline Punakallio et al $2004^{22}$ & 135 fire-fighters $(M)$, mean & Decrease in WAI & Poor functional balance & OR $2.4(0.9$ to 5.4$)$ & $1 \mathrm{~A}$ \\
\hline & age 40.7 years & classification & Poor sway velocity $(\mathrm{mm} / \mathrm{s})$ & OR 1.8 (0.7 to 4.5$)$ & $1 \mathrm{~A}$ \\
\hline & & & Poor mean amplitude (mm) & OR $1.3(0.5$ to 3.2$)$ & $1 \mathrm{~A}$ \\
\hline & & & $\begin{array}{l}\text { Poor-to-moderate perceived } \\
\text { balance }\end{array}$ & OR 9.8 (3.8 to 24.9 ) & $1 \mathrm{~A}$ \\
\hline Sjogren-Ronka et al & 88 office workers, mean age & WAI 7-49 & Good spine forward flexion & $\beta 0.24$ (Sign.) & $2 \mathrm{~A}, \mathrm{C} 3 \mathrm{~B}$ \\
\hline $2002^{23}$ & 45.7 years & & High self-confidence & $\beta 0.29$ (Sign.) & $2 A, B, C 3 B$ \\
\hline Tuomi et al $2001^{25}$ & $\begin{array}{l}1101 \text { Finnish active workers, } \\
\text { mean age } 58.4 \text { years }\end{array}$ & WAI 7-49 & $\begin{array}{l}\text { Physical exercise during free } \\
\text { time }\end{array}$ & $\beta 1.07$ (Sign.) & $3 C$ \\
\hline & & & Alcohol drinking (yes/no) & $\beta 1.53$ (Sign.) & $3 \mathrm{~B}, \mathrm{C}$ \\
\hline & & & BMI $\left(\mathrm{kg} / \mathrm{m}^{2}\right)$ & $\beta-0.78$ (Sign.) & $3 B, C$ \\
\hline & & & Smoking & $\beta 0.57$ (p 0.30) & 3B \\
\hline
\end{tabular}

${ }^{*}$ For the identification of the covariates, see table 6 .

$\beta$, linear regression coefficient; BMI, body mass index; $F$, females; $L T$, leisure time; $M$, males; OR, odds ratio; Sign., $p<0.05$; V0 2 max, maximum oxygen consumption.

may be argued that job training is not a causal factor for poor WAI, but that workers with a poor WAI were likely to have received job retraining in order to increase their work ability.

Another limitation lies in the nature of the synthesis of results. A meta-analysis was not possible, because of the large heterogeneity in definition and measurement of determinants.
For example, musculoskeletal capacity was characterised from a poor trunk muscular endurance to good spine forward flexion. Although the review was limited to studies using the same measurement method for work ability, comparability was hampered by differences in outcome definition (WAI as linear variable vs dichotomised for poor work ability with different 
Table 3 Associations between individual determinants and Work Ability Index (WAI) in longitudinal epidemiological studies among occupational populations

\begin{tabular}{|c|c|c|c|c|c|c|}
\hline Authors & Follow-up & Study population & WAI outcome & Determinant & $\begin{array}{l}\text { Measure of association } \\
(95 \% \mathrm{CI})\end{array}$ & Adjustments* \\
\hline \multirow[t]{6}{*}{ Laitinen et al $2005^{16}$} & \multirow{6}{*}{$\begin{array}{l}\mathrm{L}\left(17 \text { years) } \mathrm{T}_{1}:\right. \\
1997\end{array}$} & \multirow{3}{*}{$\begin{array}{l}2674 \text { Finnish workers } \\
\text { (M), mean age } 14 \\
\text { years }\end{array}$} & \multirow[t]{3}{*}{ Poor WAI (lowest 15\%) } & $\mathrm{BMI} \geqslant 24.5 \mathrm{~kg} / \mathrm{m}^{2}$ & OR 1.5 (0.8 to 3.0$)$ & $1 \mathrm{C}, 3 \mathrm{C}$ \\
\hline & & & & $\mathrm{BMI}<15.49 \mathrm{~kg} / \mathrm{m}^{2}$ & OR 2.2 (1.1 to 4.7$)$ & $1 \mathrm{C}, 3 \mathrm{C}$ \\
\hline & & & & $\begin{array}{l}\text { Always overweight during } \\
\text { follow-up }\end{array}$ & OR 0.8 (0.6 to 1.2$)$ & $1 \mathrm{C}, 3 \mathrm{C}$ \\
\hline & & \multirow{3}{*}{$\begin{array}{l}2948 \text { Finnish workers } \\
(\mathrm{F}) \text {, mean age } 14 \\
\text { years }\end{array}$} & \multirow[t]{3}{*}{ Poor WAI (lowest 15\%) } & $\mathrm{BMI} \geqslant 24.5 \mathrm{~kg} / \mathrm{m}^{2}$ & OR 2.0 (1.1 to 3.2 ) & $1 \mathrm{C}, 3 \mathrm{C}$ \\
\hline & & & & $\mathrm{BMI}<15.49 \mathrm{~kg} / \mathrm{m}^{2}$ & OR 2.8 (1.6 to 5.1$)$ & $1 \mathrm{C}, 3 \mathrm{C}$ \\
\hline & & & & $\begin{array}{l}\text { Always overweight during } \\
\text { follow-up }\end{array}$ & OR 1.4 (1.0 to 2.0$)$ & $1 \mathrm{C}, 3 \mathrm{C}$ \\
\hline \multirow[t]{4}{*}{ Pohjonen $2001^{19}$} & \multirow{4}{*}{$\begin{array}{l}L(5 \text { years) } \\
1993-1998\end{array}$} & \multirow{4}{*}{$\begin{array}{l}132 \text { home care } \\
\text { workers (F), mean } \\
\text { age } 41 \text { years }\end{array}$} & \multirow{4}{*}{$\begin{array}{l}\text { Decrease WAI } \\
\text { classification }\end{array}$} & Poor balance & OR 6.53 (1.84 to 23.25$)$ & $1 \mathrm{~A}, \mathrm{~T}_{0}$ \\
\hline & & & & Poor sit-up & OR 8.88 (2.42 to 32.60$)$ & $1 \mathrm{~A}, \mathrm{~T}_{0}$ \\
\hline & & & & Poor $\mathrm{VO}_{2 \max }(\mathrm{ml} / \mathrm{min} / \mathrm{kg})$ & OR 1.94 (0.44 to 8.54$)$ & $1 \mathrm{~A}, \mathrm{~T}_{0}$ \\
\hline & & & & $\mathrm{BMI} \geqslant 30 \mathrm{~kg} / \mathrm{m}^{2}$ & OR 7.51 (1.88 to 30.0 ) & $1 \mathrm{~A}, \mathrm{~T}_{0}$ \\
\hline \multirow{3}{*}{$\begin{array}{l}\text { Punakallio et al } \\
2004^{22}\end{array}$} & \multirow{3}{*}{$\begin{array}{l}\text { L (3 years) } \\
1996-1999\end{array}$} & \multirow{3}{*}{$\begin{array}{l}135 \text { fire-fighters }(\mathrm{M}) \text {, } \\
\text { mean age } 40.7 \text { years }\end{array}$} & \multirow{3}{*}{$\begin{array}{l}\text { Decrease WAI } \\
\text { classification }\end{array}$} & Poor functional balance & OR 3.6 (1.0 to 12.7 ) & $1 \mathrm{~A}, \mathrm{~T}_{0}$ \\
\hline & & & & Poor sway amplitude (mm) & OR 2.3 (0.9 to 6.1$)$ & $1 \mathrm{~A}, \mathrm{~T}_{0}$ \\
\hline & & & & $\begin{array}{l}\text { Poor-to-moderate perceived } \\
\text { balance }\end{array}$ & OR 2.4 (0.9 to 6.6$)$ & $1 \mathrm{~A}, \mathrm{~T}_{0}$ \\
\hline \multirow[t]{15}{*}{ Tuomi et al $1991^{24}$} & \multirow{15}{*}{$\begin{array}{l}\mathrm{L}(4 \text { years) } \\
1981-1985\end{array}$} & \multirow{3}{*}{$\begin{array}{l}4255 \text { municipal } \\
\text { workers, mean age } \\
50 \text { years }\end{array}$} & \multirow[t]{15}{*}{ Decrease in WAI } & Age (years) & $\beta-0.10$ (Sign.) & $1 \mathrm{~A} 3 \mathrm{~A}, \mathrm{C} 4 \mathrm{~A}, \mathrm{C} 5 \mathrm{~A}, \mathrm{~B}$ \\
\hline & & & & BMI $\left(\mathrm{kg} / \mathrm{m}^{2}\right)$ & $\beta-0.05$ (Sign.) & $1 \mathrm{~A} 3 \mathrm{~A}, \mathrm{C} 4 \mathrm{~A}, \mathrm{C} 5 \mathrm{~A}, \mathrm{~B}$ \\
\hline & & & & Cigarette smoker & $\beta-0.04$ (Sign.) & $1 \mathrm{~A} 3 \mathrm{~A}, \mathrm{C} 4 \mathrm{~A}, \mathrm{C} 5 \mathrm{~A}, \mathrm{~B}$ \\
\hline & & \multirow{5}{*}{$\begin{array}{l}1064 \text { municipal } \\
\text { workers with } \\
\text { musculoskeletal } \\
\text { disease }\end{array}$} & & Marital status (no/yes) & $\beta-0.06$ (Sign.) & $1 \mathrm{~A} 3 \mathrm{~A}, \mathrm{C} 4 \mathrm{~A}, \mathrm{C} 5 \mathrm{~A}, \mathrm{~B}$ \\
\hline & & & & Low basic education & $\beta-0.15$ (Sign.) & $1 \mathrm{~A} 3 \mathrm{~A}, \mathrm{C} 4 \mathrm{~A}, \mathrm{C} 5 \mathrm{~A}, \mathrm{~B}$ \\
\hline & & & & Life satisfaction & $\beta 0.11$ (Sign.) & $1 \mathrm{~A} 3 \mathrm{~A}, \mathrm{C} 4 \mathrm{~A}, \mathrm{C} 5 \mathrm{~A}, \mathrm{~B}$ \\
\hline & & & & Physical exercise & $\beta 0.08$ (Sign.) & $1 \mathrm{~A} 3 \mathrm{~A}, \mathrm{C} 4 \mathrm{~A}, \mathrm{C} 5 \mathrm{~A}, \mathrm{~B}$ \\
\hline & & & & Age (years) & $\beta-0.13$ (Sign.) & $1 \mathrm{~A} 3 \mathrm{~A}, \mathrm{C} 4 \mathrm{~A}, \mathrm{C} 5 \mathrm{~A}, \mathrm{~B}$ \\
\hline & & \multirow{4}{*}{$\begin{array}{l}522 \text { municipal } \\
\text { workers with } \\
\text { cardiovascular } \\
\text { disease }\end{array}$} & & Low basic education & $\beta-0.16$ (Sign.) & $1 \mathrm{~A} 3 \mathrm{~A}, \mathrm{C} 4 \mathrm{~A}, \mathrm{C} 5 \mathrm{~A}, \mathrm{~B}$ \\
\hline & & & & Life satisfaction & $\beta 0.19$ (Sign.) & $1 \mathrm{~A} 3 \mathrm{~A}, \mathrm{C} 4 \mathrm{~A}, \mathrm{C} 5 \mathrm{~A}, \mathrm{~B}$ \\
\hline & & & & Physical exercise & $\beta 0.10$ (Sign.) & $1 \mathrm{~A} 3 \mathrm{~A}, \mathrm{C} 4 \mathrm{~A}, \mathrm{C} 5 \mathrm{~A}, \mathrm{~B}$ \\
\hline & & & & Age (years) & $\beta-0.09$ (Sign.) & $1 \mathrm{~A} 3 \mathrm{~A}, \mathrm{C} 4 \mathrm{~A}, \mathrm{C} 5 \mathrm{~A}, \mathrm{~B}$ \\
\hline & & \multirow{3}{*}{$\begin{array}{l}118 \text { municipal } \\
\text { workers with mental } \\
\text { disease }\end{array}$} & & Low basic education & $\beta-0.19$ (Sign.) & $1 \mathrm{~A} 3 \mathrm{~A}, \mathrm{C} 4 \mathrm{~A}, \mathrm{C} 5 \mathrm{~A}, \mathrm{~B}$ \\
\hline & & & & Life satisfaction & $\beta 0.23$ (Sign.) & $1 \mathrm{~A} 3 \mathrm{~A}, \mathrm{C} 4 \mathrm{~A}, \mathrm{C} 5 \mathrm{~A}, \mathrm{~B}$ \\
\hline & & & & Age (years) & $\beta-0.16$ (Sign.) & $1 \mathrm{~A} 3 \mathrm{~A}, \mathrm{C} 4 \mathrm{~A}, \mathrm{C} 5 \mathrm{~A}, \mathrm{~B}$ \\
\hline \multirow[t]{4}{*}{ Tuomi et al $1997^{26}$} & \multirow{4}{*}{$\begin{array}{l}\text { L (11 years) } \\
1981-1992\end{array}$} & \multirow{4}{*}{$\begin{array}{l}818 \text { municipal } \\
\text { workers, mean age } \\
47 \text { years }\end{array}$} & Increase $(\geqslant 3)$ in WAI & Age (years) & OR $1.0(0.9$ to 1.2$)$ & $1 \mathrm{~A} 3 \mathrm{~B} 4 \mathrm{~B} 5 \mathrm{~A}, \mathrm{~B}$ \\
\hline & & & Decline $(\geqslant 10)$ in WAI & Age (years) & OR 1.1 (1.0 to 1.2$)$ & $1 \mathrm{~A} 3 \mathrm{~B} 4 \mathrm{~B} 5 \mathrm{~A}, \mathrm{~B}$ \\
\hline & & & Increase $(\geqslant 3)$ in WAI & $\begin{array}{l}\text { Increased vigorous physical } \\
\text { exercise }\end{array}$ & OR 1.8 (1.0 to 3.5$)$ & $5 A, 4 C$ \\
\hline & & & Decline $(\geqslant 10)$ in WAI & $\begin{array}{l}\text { Decreased vigorous physical } \\
\text { exercise }\end{array}$ & OR 1.8 (1.2 to 2.8$)$ & $5 A, B 4 C$ \\
\hline \multirow[t]{2}{*}{ Tuomi et al $2004^{27}$} & \multirow[t]{2}{*}{$\begin{array}{l}L(2 \text { years }) \\
1998-2000\end{array}$} & \multirow{2}{*}{$\begin{array}{l}1389 \text { metal and retail } \\
\text { workers, mean age } \\
43.9 \text { years }\end{array}$} & \multirow[t]{2}{*}{ Increase in WAI } & $\begin{array}{l}\text { Improvements in economic } \\
\text { position }\end{array}$ & $\beta 0.53$ (Sign.) & $\begin{array}{l}1 \mathrm{~A}, \mathrm{~B} 3 \mathrm{~B}, \mathrm{C} 4 \mathrm{~A}, \mathrm{~B}, \mathrm{C} \\
5 \mathrm{~A}, \mathrm{~B}\end{array}$ \\
\hline & & & & Increase in physical exercise & $\beta 0.29$ (Sign.) & $\begin{array}{l}1 \mathrm{~A}, \mathrm{~B} 3 \mathrm{C} 4 \mathrm{~A}, \mathrm{~B}, \mathrm{C} 5 \mathrm{~A} \\
\mathrm{~B}\end{array}$ \\
\hline
\end{tabular}

\footnotetext{
${ }^{*}$ For the identification of the covariates, see table 6 .
}

$\beta$, linear regression coefficient; $B M I$, body mass index; $F$, females; $L$, longitudinal study design; $M$, males; $0 R$, odds ratio; Sign., $p<0.05$; $T_{0}$, baseline results; $\mathrm{V}_{2 \text { max }}$, maximum oxygen consumption.

cut-offs). Besides, studies with different study designs (crosssectional vs longitudinal) were included.

The selected studies were dominated by Finnish studies (70\%), with also heavy emphasis on research among municipal workers. Therefore, some caution is needed in the generalisibility of the study results to other occupational populations in other countries.

\section{Interpretation of null associations}

This review not only described individual and work-related determinants associated with a poor WAI, but also evaluated negative and null associations (table 6). The number of null associations was independent of type of determinant and study design. A null association may be the result of (a) a small sample size and lack of statistical power; (b) lack of exposure variability; (c) presence of another risk factor or confounder; and (d) non-differential measurement error. The first reason for an inconclusive result, a small sample size, may explain the nonsignificant associations for cardiorespiratory capacity, ${ }^{13} 19$ overweight, ${ }^{28}$ and poor functional balance ${ }^{22}$ in study populations with less than 100 subjects. Similarly, a definition of a body mass index $\geqslant 35$ will probably not give a sufficient number of cases for a meaningful analysis ${ }^{16}$ Lack of exposure variability could be another explanation for null associations. For example, when the population was restricted to workers older than 40 years or workers within the same occupation, the population will be more homogenous and, hence, will have limited contrast in age and work-related determinants and, thus, their influence on WAI will be difficult to determine. ${ }^{24}$ The presence of another risk factor or confounder seems a likely explanation for the null associations in studies not controlled for confounders. ${ }^{13} 29$ Finally, a null association could also be due to substantial non-differential misclassification in the determinants. Misclassification is especially expected in self-reported measures with limited answer categories, for example leisure-time 
Table 4 Associations between work-related determinants and Work Ability Index (WAI) in cross-sectional epidemiological studies among occupational populations

\begin{tabular}{|c|c|c|c|c|c|}
\hline Authors & Study population & WAI & Determinant & $\begin{array}{l}\text { Measure of association } \\
(95 \% \mathrm{CI})\end{array}$ & Adjustments* \\
\hline \multirow[t]{2}{*}{ Aittomaki et al $2003^{12}$} & $\begin{array}{l}429 \text { municipal workers (M), mean } \\
\text { age } 49.2 \text { years }\end{array}$ & WAI $<32$ & Blue-collar worker & OR 0.95 (0.42 to 2.19$)$ & $1 \mathrm{~A}, \mathrm{E} 4 \mathrm{C}$ \\
\hline & $\begin{array}{l}1398 \text { municipal workers }(F) \text {, mean } \\
\text { age } 49.2 \text { years }\end{array}$ & WAI $<32$ & Blue-collar worker & OR $1.11(0.67$ to 1.84$)$ & $1 \mathrm{~A}, \mathrm{E} 4 \mathrm{C}$ \\
\hline \multirow[t]{7}{*}{ Fischer et al $2006^{14}$} & \multirow{7}{*}{$\begin{array}{l}696 \text { care givers, mean age } 34.9 \\
\text { years }\end{array}$} & \multirow[t]{7}{*}{ WAI $<37$} & Shift work & OR 1.61 (0.89 to 2.91$)$ & $1 A, D, E 3 A 4 C 5 A, B$ \\
\hline & & & Never organised workplace & OR 0.69 (0.32 to 1.51$)$ & $1 A, D, E 3 A 4 C 5 A, B$ \\
\hline & & & Often conflict with patients & OR 1.39 (0.82 to 2.35$)$ & $1 A, D, E 3 A 4 C 5 A, B$ \\
\hline & & & $\begin{array}{l}>2 \text { times verbal abuse (past } \\
\text { month) }\end{array}$ & OR 1.67 (1.00 to 3.04$)$ & $1 A, D, E 3 A 4 C 5 A, B$ \\
\hline & & & High-strain job & OR $1.21(0.70$ to 2.10$)$ & $1 A, D, E 3 A 4 C 5 A, B$ \\
\hline & & & Often thermal discomfort & OR 1.55 (1.00 to 2.40$)$ & $1 A, D, E 3 A 4 C 5 A, B$ \\
\hline & & & Often lifting patients & OR 2.02 (0.96 to 4.25$)$ & $1 A, D, E 3 A 4 C 5 A, B$ \\
\hline \multirow[t]{5}{*}{ Pohjonen $2001 b^{20}$} & \multirow{5}{*}{$\begin{array}{l}636 \text { home care workers }(F) \text {, mean } \\
\text { age } 42.3 \text { years }\end{array}$} & \multirow{5}{*}{$\begin{array}{l}\text { Decrease WAI } \\
\text { classification }\end{array}$} & High time pressure & OR 1.05 (0.53 to 2.07$)$ & $4 A, B, C 5 A$ \\
\hline & & & $\begin{array}{l}\text { Poor possibilities to control one's } \\
\text { own work }\end{array}$ & OR 1.95 (1.02 to 3.72$)$ & $1 A, C, E 3 B 4 A, B, C 5 A$ \\
\hline & & & Poor management & OR 1.58 (0.86 to 2.94$)$ & $4 A, B, C 5 A$ \\
\hline & & & High mental work demands & OR 1.40 (0.79 to 2.48$)$ & None \\
\hline & & & Poor ergonomic conditions & OR 2.54 (1.21 to 5.30$)$ & $1 \mathrm{~A}, \mathrm{C}, \mathrm{E} 3 \mathrm{~B} 4 \mathrm{~A}, \mathrm{~B}, \mathrm{C} 5 \mathrm{~A}$ \\
\hline Pranjic et al $2006^{21}$ & 534 physicians, mean age 44 years & $\begin{array}{l}\text { Decrease WAI } \\
\text { classification }\end{array}$ & Often exposed to mobbing & OR 4.75 (4.14 to 5.35$)$ & $4 \mathrm{C}$ \\
\hline $\begin{array}{l}\text { Sjogren-Ronka et al } \\
2002^{23}\end{array}$ & $\begin{array}{l}88 \text { office workers, mean age } 45.7 \\
\text { years }\end{array}$ & WAI 7-49 & High mental stress & $\beta-0.17$ (Sign.) & $2 A, B, C 3 B$ \\
\hline \multirow[t]{15}{*}{ Tuomi et al $2001^{25}$} & \multirow{15}{*}{$\begin{array}{l}1101 \text { Finnish active workers, mean } \\
\text { age } 58.4 \text { years }\end{array}$} & \multirow[t]{15}{*}{ WAI 7-49 } & Muscular work & $\beta-0.22(0.067)$ & $4 A, C 5 A, B$ \\
\hline & & & Poor work postures & $\beta-0.44$ (Sign.) & $4 A, C 5 A, B$ \\
\hline & & & Intelligence demand & $\beta 0.46$ (Sign.) & $4 A, C 5 A, B$ \\
\hline & & & Poor work tools and rooms & $\beta-0.35$ (Sign.) & $4 A, C 5 A, B$ \\
\hline & & & Poor physical climate & $\beta-0.29$ (Sign.) & $4 A, C 5 A, B$ \\
\hline & & & Restless work environment & $\beta-0.33$ (Sign.) & $4 A, C 5 A, B$ \\
\hline & & & Poor management & $\beta-0.54$ (Sign.) & $4 B, C$ \\
\hline & & & Lack of freedom & $\beta-0.31$ (Sign.) & $4 B, C$ \\
\hline & & & Uninspiring work & $\beta-0.65$ (Sign.) & $4 \mathrm{~B}, \mathrm{C}$ \\
\hline & & & Utilisation of work experience & $\beta 0.94$ (Sign.) & $4 B, C$ \\
\hline & & & $\begin{array}{l}\text { Possibilities for development and } \\
\text { influence at work }\end{array}$ & $\beta 0.65$ (Sign.) & $4 \mathrm{C}$ \\
\hline & & & Job retraining & $\beta-3.41$ (Sign.) & $4 \mathrm{C}$ \\
\hline & & & $\begin{array}{l}\text { Subjective improvement in work } \\
\text { and tasks }\end{array}$ & ß 1.05 (Sign.) & $4 A, C 5 A, B$ \\
\hline & & & $\begin{array}{l}\text { Subjective improvement in work } \\
\text { environment and tools }\end{array}$ & $\beta 0.47(0.089)$ & $4 A, C 5 A, B$ \\
\hline & & & $\begin{array}{l}\text { Subjective increase in mental } \\
\text { workload }\end{array}$ & $\beta-1.21$ (Sign.) & $4 A, C 5 A, B$ \\
\hline
\end{tabular}

${ }^{*}$ For the identification of the covariates, see table 6 .

$\beta$, linear regression coefficient; $F$, females; $M$, males; $O R$, odds ratio; Sign., $p<0.05$.

physical activity in three levels of frequency per week. ${ }^{20}$ The quality assessment indeed showed most studies lost points because substantial misclassification in determinants was likely to occur. The total quality score however showed no differences for type of determinant or significance of reported associations.

\section{Individual determinants}

For individual determinants the range in magnitude of associations was larger in cross-sectional studies than in longitudinal studies. A cross-sectional study design is more sensitive to bias, which may explain the larger differences in measure of association. For some determinants the available number of studies was too small to draw meaningful conclusions, that is, for gender (two studies), education (two studies), and mental performance (two studies). For other determinants the number of significant associations equaled the number of null associations, for example overweight (four positive associations against three null associations).
In one study a negative association was found between older age ( $\geqslant 40$ year) and poor WAI. ${ }^{14}$ The study population consisted mainly of female healthcare workers with an age below 35 years, hence, the negative association is most likely due to a strong "healthy worker selection effect". Another negative association was found for alcohol drinking. ${ }^{25}$ This association may have been the result of the fact that the effects of problematic alcohol use were not evaluated separately, whereas moderate alcohol has beneficial effects on health.

\section{Work-related determinants}

Despite the large differences in definition of the determinants and the validity of the measurement techniques applied, the studies consistently showed that important determinants for WAI were high mental work demands, poor autonomy, and high physical work demands. A recent study also demonstrated significant associations between these work-related determinants and work ability. ${ }^{32}$ 
Table 5 Associations between work-related determinants and Work Ability Index (WAI) in longitudinal epidemiological studies among occupational populations

\begin{tabular}{|c|c|c|c|c|c|c|}
\hline Authors & Follow-up & Study population & WAI outcome & Determinant & $\begin{array}{l}\text { Measure of association } \\
(95 \% \mathrm{Cl})\end{array}$ & Adjustments* \\
\hline \multirow{20}{*}{$\begin{array}{l}\text { Tuomi et al } \\
1991^{24}\end{array}$} & \multirow{20}{*}{$\begin{array}{l}L(4 \text { years) } \\
1981-1985\end{array}$} & \multirow{5}{*}{$\begin{array}{l}4255 \text { municipal workers, mean age } \\
50 \text { years }\end{array}$} & \multirow[t]{5}{*}{ Change in WAI } & High physical demands & $\beta-0.06$ (Sign.) & $1 \mathrm{~A} 3 \mathrm{~A}, \mathrm{C} 4 \mathrm{~A}, \mathrm{C} 5 \mathrm{~A}, \mathrm{~B}$ \\
\hline & & & & $\begin{array}{l}\text { Good possibilities to } \\
\text { develop }\end{array}$ & $\beta 0.03$ (Sign.) & $1 \mathrm{~A} 3 \mathrm{~A}, \mathrm{C} 4 \mathrm{~A}, \mathrm{C} 5 \mathrm{~A}, \mathrm{~B}$ \\
\hline & & & & $\begin{array}{l}\text { Poor physical } \\
\text { environment }\end{array}$ & $\beta-0.05$ (Sign.) & $1 \mathrm{~A} 3 \mathrm{~A}, \mathrm{C} 4 \mathrm{~A}, \mathrm{C} 5 \mathrm{~A}, \mathrm{~B}$ \\
\hline & & & & Poor work schedule & $\beta-0.03$ (Sign.) & $1 \mathrm{~A} 3 \mathrm{~A}, \mathrm{C} 4 \mathrm{~A}, \mathrm{C} 5 \mathrm{~A}, \mathrm{~B}$ \\
\hline & & & & Physical stress at work & $\beta-0.08$ (Sign.) & $1 \mathrm{~A} 3 \mathrm{~A}, \mathrm{C} 4 \mathrm{~A}, \mathrm{C} 5 \mathrm{~A}, \mathrm{~B}$ \\
\hline & & \multirow{7}{*}{$\begin{array}{l}1064 \text { municipal workers with } \\
\text { musculoskeletal disease }\end{array}$} & \multirow[t]{7}{*}{ Change in WAI } & Muscular work & $\beta-0.09$ (Sign.) & $1 \mathrm{~A} 3 \mathrm{~A}, \mathrm{C} 4 \mathrm{~A}, \mathrm{C} 5 \mathrm{~A}, \mathrm{~B}$ \\
\hline & & & & Poor work posture & $\beta-0.10$ (Sign.) & $1 \mathrm{~A} 3 \mathrm{~A}, \mathrm{C} 4 \mathrm{~A}, \mathrm{C} 5 \mathrm{~A}, \mathrm{~B}$ \\
\hline & & & & $\begin{array}{l}\text { Change of workload in } \\
\text { the past } 2 \text { years }\end{array}$ & $\beta-0.07$ (Sign.) & $1 \mathrm{~A} 3 \mathrm{~A}, \mathrm{C} 4 \mathrm{~A}, \mathrm{C} 5 \mathrm{~A}, \mathrm{~B}$ \\
\hline & & & & Poor work temperature & $\beta-0.16$ (Sign.) & $1 \mathrm{~A} 3 \mathrm{~A}, \mathrm{C} 4 \mathrm{~A}, \mathrm{C} 5 \mathrm{~A}, \mathrm{~B}$ \\
\hline & & & & Poor management & $\beta-0.10$ (Sign.) & $1 \mathrm{~A} 3 \mathrm{~A}, \mathrm{C} 4 \mathrm{~A}, \mathrm{C} 5 \mathrm{~A}, \mathrm{~B}$ \\
\hline & & & & Lack of freedom & $\beta-0.07$ (Sign.) & $1 \mathrm{~A} 3 \mathrm{~A}, \mathrm{C} 4 \mathrm{~A}, \mathrm{C} 5 \mathrm{~A}, \mathrm{~B}$ \\
\hline & & & & Poor work schedule & $\beta-0.08$ (Sign.) & $1 \mathrm{~A} 3 \mathrm{~A}, \mathrm{C} 4 \mathrm{~A}, \mathrm{C} 5 \mathrm{~A}, \mathrm{~B}$ \\
\hline & & \multirow{7}{*}{$\begin{array}{l}522 \text { municipal workers with } \\
\text { cardiovascular disease }\end{array}$} & \multirow[t]{7}{*}{ Change in WAI } & Muscular work & $\beta-0.27$ (Sign.) & $1 \mathrm{~A} 3 \mathrm{~A}, \mathrm{C} 4 \mathrm{~A}, \mathrm{C} 5 \mathrm{~A}, \mathrm{~B}$ \\
\hline & & & & Sitting work & $\beta-0.15$ (Sign.) & $1 \mathrm{~A} 3 \mathrm{~A}, \mathrm{C} 4 \mathrm{~A}, \mathrm{C} 5 \mathrm{~A}, \mathrm{~B}$ \\
\hline & & & & $\begin{array}{l}\text { Responsibility for } \\
\text { people }\end{array}$ & $\beta 0.10$ (Sign.) & $1 \mathrm{~A} 3 \mathrm{~A}, \mathrm{C} 4 \mathrm{~A}, \mathrm{C} 5 \mathrm{~A}, \mathrm{~B}$ \\
\hline & & & & Poor tools and rooms & $\beta-0.08$ (Sign.) & $1 \mathrm{~A} 3 \mathrm{~A}, \mathrm{C} 4 \mathrm{~A}, \mathrm{C} 5 \mathrm{~A}, \mathrm{~B}$ \\
\hline & & & & Poor physical climate & $\beta-0.10$ (Sign.) & $1 \mathrm{~A} 3 \mathrm{~A}, \mathrm{C} 4 \mathrm{~A}, \mathrm{C} 5 \mathrm{~A}, \mathrm{~B}$ \\
\hline & & & & $\begin{array}{l}\text { Noisy and restless } \\
\text { workplace }\end{array}$ & $\beta-0.09$ (Sign.) & $1 \mathrm{~A} 3 \mathrm{~A}, \mathrm{C} 4 \mathrm{~A}, \mathrm{C} 5 \mathrm{~A}, \mathrm{~B}$ \\
\hline & & & & Poor work schedule & $\beta-0.07$ (Sign.) & $1 \mathrm{~A} 3 \mathrm{~A}, \mathrm{C} 4 \mathrm{~A}, \mathrm{C} 5 \mathrm{~A}, \mathrm{~B}$ \\
\hline & & $\begin{array}{l}118 \text { municipal workers with mental } \\
\text { disease }\end{array}$ & Change in WAI & Lack of freedom & $\beta-0.27$ (Sign.) & $1 \mathrm{~A} 3 \mathrm{~A}, \mathrm{C} 4 \mathrm{~A}, \mathrm{C} 5 \mathrm{~A}, \mathrm{~B}$ \\
\hline \multirow[t]{13}{*}{$\begin{array}{l}\text { Tuomi et al } \\
1997^{26}\end{array}$} & \multirow[t]{13}{*}{$\begin{array}{l}\mathrm{L}(11 \text { years) } \\
1981-1992\end{array}$} & \multirow[t]{13}{*}{$\begin{array}{l}818 \text { municipal workers, mean age } 47 \\
\text { years }\end{array}$} & \multirow[t]{6}{*}{ Increase $(+\geqslant 3)$ in WAI } & $\begin{array}{l}\text { No harmful lack of } \\
\text { freedom }\end{array}$ & OR 0.9 (0.8 to 1.0$)$ & None \\
\hline & & & & $\begin{array}{l}\text { Decreased noisy and } \\
\text { restless workplace }\end{array}$ & OR 3.4 (1.6 to 7.2 ) & $1 \mathrm{~A} 3 \mathrm{~B} 4 \mathrm{~B}, \mathrm{C} 5 \mathrm{~A}$ \\
\hline & & & & $\begin{array}{l}\text { Decreased } \\
\text { management strain }\end{array}$ & OR $2.0(1.0$ to 3.7$)$ & $1 \mathrm{~A} 3 \mathrm{~B} 4 \mathrm{~B}, \mathrm{C} 5 \mathrm{~A}$ \\
\hline & & & & $\begin{array}{l}\text { Decreased role } \\
\text { ambiguity }\end{array}$ & OR 2.1 (0.9 to 5.1$)$ & None \\
\hline & & & & Increased freedom & OR 2.8 (1.0 to 7.8 ) & None \\
\hline & & & & $\begin{array}{l}\text { Increased satisfaction } \\
\text { with supervisor's } \\
\text { attitude }\end{array}$ & OR 3.6 (1.8 to 7.2 ) & $3 B, 5 A$ \\
\hline & & & \multirow[t]{4}{*}{ Decrease $(-\geqslant 10)$} & $\begin{array}{l}\text { Decreased possibilities } \\
\text { for development and } \\
\text { influence at work }\end{array}$ & OR 2.4 (1.4 to 4.3 ) & $1 \mathrm{~A} 3 \mathrm{~B} 4 \mathrm{~B}, \mathrm{C} 5 \mathrm{~A}, \mathrm{~B}$ \\
\hline & & & & $\begin{array}{l}\text { Increased role } \\
\text { ambiguity }\end{array}$ & OR 1.9 (1.4 to 2.7$)$ & $1 \mathrm{~A} 3 \mathrm{~B} 4 \mathrm{~B}, \mathrm{C} 5 \mathrm{~A}, \mathrm{~B}$ \\
\hline & & & & Decreased freedom & OR 1.4 (1.0 to 2.0$)$ & None \\
\hline & & & & $\begin{array}{l}\text { Decreased possibility } \\
\text { for recognition and } \\
\text { esteem at work }\end{array}$ & OR 2.4 (1.4 to 4.3$)$ & $3 B 5 A, B$ \\
\hline & & & \multirow[t]{3}{*}{ Increase $(+\geqslant 3)$ in WAI } & $\begin{array}{l}\text { Decreased muscular } \\
\text { work }\end{array}$ & OR 2.8 (1.2 to 6.6$)$ & None \\
\hline & & & & $\begin{array}{l}\text { Improved work } \\
\text { postures }\end{array}$ & OR 2.9 (1.3 to 6.5$)$ & $1 \mathrm{~A} 3 \mathrm{~B} 4 \mathrm{~B}, \mathrm{C} 5 \mathrm{~A}$ \\
\hline & & & & $\begin{array}{l}\text { Decreased repetitive } \\
\text { movements }\end{array}$ & OR 2.1 (1.0 to 3.4$)$ & $3 B, 4 C$ \\
\hline \multirow{4}{*}{$\begin{array}{l}\text { Tuomi et al } \\
1997^{26}\end{array}$} & \multirow{4}{*}{$\begin{array}{l}\mathrm{L}(11 \text { years }) \\
1981-1992\end{array}$} & \multirow{4}{*}{$\begin{array}{l}818 \text { municipal workers, mean age } 47 \\
\text { years }\end{array}$} & \multirow[t]{4}{*}{ Decrease $(-\geqslant 10)$} & Poor work temperature & OR 1.1 (1.0 to 1.1$)$ & $3 B 5 A, B$ \\
\hline & & & & $\begin{array}{l}\text { Increased muscular } \\
\text { work }\end{array}$ & OR 1.8 (1.2 to 2.8$)$ & $3 B 5 A, B$ \\
\hline & & & & $\begin{array}{l}\text { Increased difficult work } \\
\text { postures }\end{array}$ & OR 1.5 (1.0 to 2.2$)$ & None \\
\hline & & & & $\begin{array}{l}\text { Increased standing in } \\
\text { one place }\end{array}$ & OR 1.7 (1.0 to 2.9$)$ & $3 B$ 4C 5A, B \\
\hline
\end{tabular}


Table 5 Continued

\begin{tabular}{|c|c|c|c|c|c|c|}
\hline Authors & Follow-up & Study population & WAI outcome & Determinant & $\begin{array}{l}\text { Measure of association } \\
(95 \% \mathrm{Cl})\end{array}$ & Adjustments* \\
\hline & & & & $\begin{array}{l}\text { Increase in promotion } \\
\text { of employee well-being }\end{array}$ & $\beta 0.53$ (Sign.) & $\begin{array}{l}1 A, B 3 B, C 4 A, B, C \\
5 A, B\end{array}$ \\
\hline & & & & $\begin{array}{l}\text { Decrease in uncertainty } \\
\text { at the workplace }\end{array}$ & $\beta 0.70$ (Sign.) & $\begin{array}{l}1 A, B 3 B, C 4 A, B, C \\
5 A, B\end{array}$ \\
\hline & & & & $\begin{array}{l}\text { Decrease in physical } \\
\text { demands at work }\end{array}$ & $\beta 1.35$ (Sign.) & $\begin{array}{l}1 A, B 3 B, C 4 A, B, C \\
5 A, B\end{array}$ \\
\hline
\end{tabular}

${ }^{*}$ For the identification of the covariates, see table 6 .

$\beta$, linear regression coefficient; $F$, females; $L$, longitudinal study design; $M$, males; $O R$, odds ratio; Sign., $p<0.05$.

All work-related determinants were measured by means of self-report. This assessment technique may lead to spurious results, when subjects with a poor WAI overestimate their physical and mental workload in the workplace relative to those with an excellent WAI. It is unclear if an objective measurement of the work demands would show similar results.

Some determinants, which feature prominently in the model of Ilmarinen, ${ }^{5}$ were not included in the observed studies. Health, functional capacity, and work were (over)represented in research, in respect to professional competence, and values, attitudes and motivation for work. Health, functional capacity and work-related risk factors have a well-studied history in the field of work and health. The influence of competence and values, attitudes, and motivation on health-related performance at work clearly lags behind. This is in agreement with the invitation of Macdonald et $a^{33}$ to incorporate work organisation into occupational health research. Besides, through increased medical standards and improvements in the work environment, it is expected that aspects of human resources management will become more important for improving work ability. This requires the development of valid measurement instruments, which until now are largely absent.

Table 6 Summary of epidemiological studies with positive and negative associations between individual and work-related factors, and poor or decreased Work Ability Index

\begin{tabular}{|c|c|c|c|c|c|c|}
\hline \multirow[b]{3}{*}{ Factor } & \multicolumn{2}{|l|}{ Positive associations } & \multicolumn{2}{|l|}{ Null associations } & \multicolumn{2}{|l|}{ Negative associations } \\
\hline & Logistic regression & Other analysis & Logistic regression & Other analysis & Logistic regression & Other analysis \\
\hline & Range OR & No & Range OR & No & Range OR & No \\
\hline
\end{tabular}

1. Demographic factors

$\begin{array}{llllllll}\text { A. Older age } & 2 & 1.90-3.57 & 2 & 2 & 0.84-1.00 & 0 & 0 \\ \text { B. Female } & 0 & & 0 & 2 & 1.30-1.43 & 0 & 0.71 \\ \text { C. Low education } & 1 & 1.20 & 0 & 1 & 1.09 & 0 & 0 \\ \text { D. Low income } & 1 & 1.92 & 1 & 1 & 0.98 & 0 & 0 \\ \text { E. Other } & 2 & 1.56-1.96 & 1 & 1 & 1.12 & 0 & 0\end{array}$

2. Physical condition

\begin{tabular}{|c|c|c|c|c|c|c|c|c|}
\hline $\begin{array}{l}\text { A. Poor cardiorespiratory } \\
\text { condition }\end{array}$ & 0 & & 1 & 2 & $1.86-1.94$ & 0 & 0 & 0 \\
\hline $\begin{array}{l}\text { B. Poor musculoskeletal } \\
\text { capacity }\end{array}$ & 3 & $6.39-9.12$ & 1 & 0 & & 0 & 0 & 0 \\
\hline C. Poor mental performance & 0 & & 0 & 2 & $1.54-1.75$ & 0 & 0 & 0 \\
\hline D. Poor balance & 1 & 6.53 & 0 & 2 & $2.40-3.60$ & 0 & 0 & 0 \\
\hline
\end{tabular}

3. Lifestyle factors

\begin{tabular}{|c|c|c|c|c|c|c|c|c|}
\hline A. Overweight & 2 & $2.71-7.51$ & 2 & 3 & $0.29-1.50$ & 0 & 0 & 0 \\
\hline $\begin{array}{l}\text { B. Lack of leisure-time } \\
\text { physical activity }\end{array}$ & 2 & $1.80-7.18$ & 2 & 1 & 1.77 & 0 & 0 & 0 \\
\hline C. Smoking & 0 & & 1 & 1 & 1.61 & 1 & 0 & 0 \\
\hline D. Other & 1 & 27.63 & 0 & 1 & 0.80 & 0 & 0 & 1 \\
\hline
\end{tabular}

4. Work-related psychosocial and organisational factors

\begin{tabular}{|c|c|c|c|c|c|c|c|c|}
\hline $\begin{array}{l}\text { A. High mental work } \\
\text { demands }\end{array}$ & 1 & 4.75 & 4 & 2 & $1.40-1.21$ & 0 & 0 & 0 \\
\hline B. Poor autonomy & 1 & 1.95 & 2 & 1 & 1.40 & 0 & 0 & 0 \\
\hline C. Other & 1 & 3.60 & 3 & 2 & $0.69-1.58$ & 0 & 0 & 1 \\
\hline
\end{tabular}

5. Work-related physical factors

\begin{tabular}{|c|c|c|c|c|c|c|c|c|}
\hline A. High physical demands & 2 & $1.80-2.54$ & 2 & 2 & $0.95-2.02$ & 1 & 0 & 0 \\
\hline B. High physical exposure & 0 & & 2 & 2 & $1.10-1.55$ & 0 & 0 & 0 \\
\hline
\end{tabular}




\section{Main messages}

- In order to increase work participation and to prolong the working life among older workers the determinants of their work ability should be studied.

- Important factors associated with a poor Work Ability Index (WAI) were lack of leisure-time vigorous physical activity, poor musculoskeletal capacity, older age, obesity, high mental work demands, lack of autonomy, poor physical work environment, and high physical work load.

- The potential impact of social and economic policies at company and national level on the WAI remain largely unknown.

\section{Implication for interventions}

This study has presented important information to consider in programmes aimed at maintaining or improving work productivity and work participation. The interventions should focus on the identified determinants associated with a lower work ability. Several work-related determinants have also been identified as important risk factors for the occurrence of sickness absence ${ }^{34}$ and for prolonged duration of sickness absence $^{35}$ and, hence, it is expected that interventions to promote maintaining or regaining a good work ability will also prevent partly (temporary) work disability. At individual level, it seems beneficial to target interventions at increasing leisuretime vigorous physical activity, increasing musculoskeletal capacity, and decreasing body mass index (i.e., obesity). Workrelated interventions should focus on an increase in autonomy at work, and decreases in physical and psychosocial demands. Professional competence and attitudes and values towards work may also be essential points of interventions in workers with decreased work ability, but their potential impact could not be demonstrated in this review.

The importance of lack of vigorous physical activity and obesity of determinants of poor work ability suggest that health promotion intervention may be beneficial. Indeed, intervention studies on increasing physical activity in leisure time and improved physical condition have shown positive effects, but were too small for a statistically significant change in the short term. ${ }^{36-39}$

Other intervention studies on work-related determinants have shown promising results. Among employees in the construction industry with a high disability risk, an assessment and individual programme for half a year focusing on optimising functioning at work showed a slight, but insignificant, improvement in WAI. ${ }^{40}$ Among farmers experiencing low back or shoulder pain, occupationally oriented rehabilitation courses including training of ergonomically correct work techniques lasting 3 weeks, showed that changes in lifting techniques were minor after 1 year's follow-up, but the WAI improved significantly for both men and women. ${ }^{41}$ Among blue-collar workers with a high disability risk, an occupational health intervention programme showed an increase in WAI, after 6 months' follow-up, yet this positive effect was not present after 2 years. ${ }^{42}$ Among truck drivers, stress management, ${ }^{39}$ and among farmers, training of work techniques ${ }^{43}$ were both not significant in changing WAI. Thus, interventions on work-related determinants have been conducted, but so far have failed to convincingly demonstrate significant improvements in WAI.

\section{Policy implications}

- In programmes aimed at maintaining and promoting the participation of the labour force, interventions should be targeted at physical workload, poor physical work environment, and psychosocial demands as well as lifestyle factors, most notably leisure-time physical activity, and body mass.

- Future research should focus on a broader perspective than work determinants at the individual or job level.

\section{Concluding remarks}

Health promotion at work can be aimed at increasing leisuretime physical activity, prevention of overweight, increasing musculoskeletal capacity and decrease of physical and psychosocial work load. This review could not demonstrate the impact of professional competences, attitudes, and work values on work ability, as defined by the WAI. In addition, factors such as the organisational context within companies and social and economic policies that influence labour participation are also lacking. Future research on determinants of work ability should incorporate the social and economic environment of workers.

Competing interests: None declared.

\section{REFERENCES}

1. IImarinen JE. Aging workers. Occup Environ Med 2001;58:546-52.

2. Stattin M. Retirement on grounds of ill health. Occup Environ Med 2005;62:135-40

3. Tuomi K, Ilmarinen J, Seitsamo J, et al. Summary of the Finnish research project (1981-1992) to promote the health and work ability of aging workers. Scand J Work Environ Health 1997;23(Suppl 1):66-71.

4. Schuring $\mathbf{M}$, Burdorf $L$, Kunst $A$, et al. The effects of ill health on entering and maintaining paid employment: evidence in European countries. J Epidemiol Community Health 2007:61:597-604.

5. IImarinen J. Towards a longer worklife. Helsinki: FIOH Bookstore, 2005.

6. Bobko NA. Work ability, age and its perception, and other related concerns of Ukraine health care workers. Exp Aging Res 2002;28:59-71.

7. Lindberg P, Vingard E, Josephson $M$, et al. Retaining the ability to work - associated factors at work. Eur J Public Health 2006;16:470-5.

8. Lindberg $\mathbf{P}$, Josephson M, Alfredsson L, et al. Promoting excellent work ability and preventing poor work ability: the same determinants? Results from the Swedish HAKuL study. Occup Environ Med 2006;63:113-20.

9. Salonen $\mathbf{P}$, Arola $\mathrm{H}$, Nygard $\mathrm{CH}$, et al. Factors associated with premature departure from working life among ageing food industry employees. Occup Med (Lond) 2003;53:65-8.

10. Burdorf A, Frings-Dresen MHW, van Duivenbooden C, et al. Development of a decision model to identify workers at risk of long-term disability in the construction industry. Scand J Work Environ Health 2005;31:31-6.

11. Tuomi K, Ilmarinen J, Jahkola A, et al. Work ability index. 2nd revised edn. Helsinki: Finnish Institute of Occupational Health, 1998.

12. Aittomaki A, Lahelma E, Roos E. Work conditions and socioeconomic inequalities in work ability. Scand J Work Environ Health 2003;29:159-65.

13. Eskelinen L, Kohvakka A, Merisalo T, et al. Relationship between the selfassessment and clinical assessment of health status and work ability. Scand J Work Environ Health 1991;17(Suppl 1):40-7.

14. Fischer FM, Borges FN, Rotenberg $L$, et al. Work ability of health care shift workers What matters? Chronobiol Int 2006;23:1165-79.

15. Goedhard WJ, Rijpstra TS, Puttiger PH. Age, absenteeism and physical fitness in relation to work ability. Stud Health Technol Inform 1998;48:254-7.

16. Laitinen J, Nayha S, Kujala V. Body mass index and weight change from adolescence into adulthood, waist-to-hip ratio and perceived work ability among young adults. Int J Obes (Lond) 2005;29:697-702.

17. Monteiro MS, Ilmarinen J, Corraa Filho HR. Work ability of workers in different age groups in a public health institution in Brazil. Int J Occup Saf Ergon 2006;12:417-27.

18. Nygard CH, Eskelinen L, Suvanto S, et al. Associations between functional capacity and work ability among elderly municipal employees. Scand J Work Environ Health 1991;17(Suppl 1):122-7.

19. Pohjonen T. Age-related physical fitness and the predictive values of fitness tests for work ability in home care work. J Occup Environ Med 2001a;43:723-30.

20. Pohjonen T. Perceived work ability of home care workers in relation to individual and work-related factors in different age groups. Occup Med (Lond) 2001b;51:209-17. 
21. Pranjic N, Males-Bilic L, Beganlic A, et al. Mobbing, stress, and work ability index among physicians in Bosnia and Herzegovina: survey study. Croat Med J 2006;47:750-8.

22. Punakallio A, Lusa S, Luukkonen R. Functional, postural and perceived balance for predicting the work ability of firefighters. Int Arch Occup Environ Health 2004;77:48290.

23. Sjogren-Ronka T, Ojanen MT, Leskinen EK, et al. Physical and psychosocial prerequisites of functioning in relation to work ability and general subjective wellbeing among office workers. Scand J Work Environ Health 2002;28:184-90.

24. Tuomi K, Eskelinen L, Toikkanen J, et al. Work load and individual factors affecting work ability among aging municipal employees. Scand J Work Environ Health 1991;17(Suppl 1):128-34

25. Tuomi K, Huuhtanen P, Nykyri E, et al. Promotion of work ability, the quality of work and retirement. Occup Med (Lond) 2001;51:318-24.

26. Tuomi K, Ilmarinen J, Martikainen R, et al. Aging, work, life-style and work ability among Finnish municipal workers in 1981-1992. Scand J Work Environ Health 1997;23(Suppl 1):58-65.

27. Tuomi K, Vanhala S, Nykyri E, et al. Organizational practices, work demands and the well-being of employees: a follow-up study in the metal industry and retail trade. Occup Med (Lond) 2004;54:115-21.

28. Kaleta D, Makowiec-Dabrowska T, Jegier A. Lifestyle index and work ability. Int J Occup Med Environ Health 2006;19:170-7.

29. Martinez MC, Latorre Mdo R. [Health and work ability among office workers] Saude e capacidade para o trabalho em trabalhadores de area administrativa. Rev Saude Publica 2006;40:851-8.

30. Dutch Cochrane Centre. Guideline for methodological quality assessment of observational studies [in Dutch]. www.cochrane.nl/downloads laccessed 1 Sep 2007).

31. Tuomi K, Huuhtanen P, Nykyri E, et al. Promotion of work ability, the quality of work and retirement. Occupational Medicine-Oxford 2001;51:318-24.

32. Alavinia SM, van Duivenbooden C, Burdorf A. Influence of work-related factors and individual characteristics on work ability among Dutch construction workers. Scand J Work Environ Health 2007;33:351-7.
33. Macdonald LA, Harenstam A, Warren ND, et al. Incorporating work organization into occupational health research: an invitation for dialogue. Occup Environ Med 2008;65:1-3.

34. Alexanderson K. Sickness absence: a review of performed studies with focused on levels of exposure and theories utilized. Scand J Soc Med 1998;26:241-9.

35. Dekkers-Sánchez PM, Hoving JL, Sluiter JK, et al. Factors associated with longterm sick leave in sicklisted employees: a systematic review. Occup Environ Med 2008;65:153-7.

36. Smolander J, Blair SN, Kohl HW 3rd. Work ability, physical activity, and cardiorespiratory fitness: 2-year results from Project Active. J Occup Environ Med 2000:42:906-10.

37. Pohjonen T, Ranta R. Effects of worksite physical exercise intervention on physical fitness, perceived health status, and work ability among home care workers: five-year follow-up. Prev Med 2001;32:465-75.

38. Nurminen E, Malmivaara A, IImarinen J, et al. Effectiveness of a worksite exercise program with respect to perceived work ability and sick leaves among women with physical work. Scand J Work Environ Health 2002;28:85-93.

39. Karazman R, Kloimuller I, Geissler $\mathrm{H}$, et al. Effects of ergonomic and health training on work interest, work ability and health in elderly public urban transport drivers. Int J Ind Erg 2000;25:503-11.

40. de Boer AG, Burdorf $A$, van Duivenbooden $C$, et al. The effect of individual counselling and education on work ability and disability pensions: a prospective intervention study in the construction industry. Occup Environ Med 2007;64:792-7.

41. Nevala-Puranen N. Effects of occupationally-oriented rehabilitation on farmers' work techniques, musculoskeletal symptoms, and work ability. J Occup Rehab 1996:6:191-200.

42. de Boer AG, van Beek JC, Durinck J, et al. An occupational health intervention programme for workers at risk for early retirement; a randomised controlled trial. Occup Environ Med 2004;61:924-9.

43. Perkio-Makela M. Influence of exercise-focused group activities on the physical activity, functional capacity, and work ability of female farmers--a three-year followup. Int J Occup Saf Ergon 1999;5:381-94

\section{Stay a step ahead with Online First}

We publish all our original articles online before they appear in a print issue. This means that the latest clinical research papers go straight from acceptance to your browser, keeping you at the cutting edge of medicine. We update the site weekly so that it remains as topical as possible. Follow the Online First link on the home page and read the latest research. 